\title{
Prenatal tobacco exposure shortens telomere length in children
}

\author{
Patrick IP ${ }^{\text {a\# }}$, Brian Hon Yin $\mathrm{CHUNG}^{\text {a\# }}$, \\ Frederick Ka Wing $\mathrm{HO}^{\mathrm{a}}$, Godfrey Chi Fung $\mathrm{CHAN}^{\mathrm{a}}$, Wen $\mathrm{DENG}^{\mathrm{b}}$, \\ Wilfred Hing Sang WONG ${ }^{\mathrm{a}}$, So Lun $\mathrm{LEE}^{\mathrm{a}}$, Purdy Ying Ting CHAN ${ }^{\mathrm{c}}$, \\ Dingge YING ${ }^{\mathrm{a}}$, Wai Lap WONGa, Keith Tsz Suen TUNG ${ }^{\mathrm{a}}$, Yu Lung LAU
}

${ }^{\#}$ Co-first authors

Affiliation: ${ }^{a}$ Department Paediatrics and Adolescent Medicine; ${ }^{b}$ School of Nursing, The University of Hong Kong; ${ }^{c}$ Child Assessment Service, Department of Health, The Government of the Hong Kong Special Administrative Region, Hong Kong, China

\section{Corresponding author:}

\section{Dr Patrick IP}

Address: Room 123, New Clinical Building, Queen Mary Hospital, Hong Kong

Telephone: (852) 28198501

Email: patricip@hku.hk 


\section{ABSTRACT (242/250 WORDS)}

Introduction: Preliminary evidence suggests a possible association between prenatal tobacco exposure and telomere length in children. This study was conducted to investigate whether maternal smoking during pregnancy was associated with telomere shortening in their children, and whether prenatal and childhood exposure to environmental tobacco had any impact on this association.

Methods: This is a population-representative study on the association between prenatal tobacco exposure and telomere length in children. 98 Hong Kong Chinese children aged under 15 years with prenatal tobacco exposure and 98 age- and gender-matched controls were recruited from a population health study with stratified random sampling.

Results: Telomere length in children with prenatal tobacco exposure was significantly shorter than in those with no exposure (mean T/S ratio $=24.9$ [SD = 8.58] in exposed vs. 28.97 [14.15] in control groups; $\mathrm{p}=0.02$ ). A negative dose-response relationship was observed between the $\mathrm{T} / \mathrm{S}$ ratio and tobacco exposure duration: the longer the duration of maternal smoking in pregnancy, the shorter the child's telomere length. The association between the child's telomere length and prenatal tobacco exposure remained significant after considering the influence of family socioeconomic status and exposure to environmental tobacco smoke during pregnancy and childhood.

Conclusions: Prenatal tobacco exposure was associated with telomere shortening in children. As this may impose significant health impacts through fetal genetic programming, more efforts should be made to reduce fetal tobacco exposure by educating pregnant women to not smoke and motivating smokers to quit in early pregnancy. 


\section{IMPLICATIONS}

As reflected by telomere shortening, prenatal tobacco exposure in children can cause premature aging and increased health risks, which we suggest is entirely preventable. Not smoking during pregnancy or quitting smoking is critical to improving the health outcome of our future generations as prenatal tobacco exposure may affect children's biological programming. 


\section{MAIN TEXT (3312 WORDS)}

\section{INTRODUCTION}

Prenatal tobacco exposure is associated with detrimental child health outcomes including premature birth, ${ }^{12}$ low birth weight, ${ }^{2-4}$ sudden infant death syndrome, ${ }^{4}$ allergic disorders, ${ }^{5-8}$ and neurodevelopmental disorders such as attention deficit hyperactivity disorder and externalizing behaviors. $^{9-11}$ In spite of these potential risks, smoking during pregnancy is still very common in China and worldwide. It is estimated that $12.8 \%$ of women in U.S. ${ }^{12}$ and $5.4 \%$ in China ${ }^{13}$ actively smoke during pregnancy.

Telomeres are specialized nucleoprotein complexes located at the distal ends of linear eukaryotic chromosomes that have critical roles in maintaining chromosomal structures during mitotic cell division by protecting the chromosomal ends from abnormal fusion and nucleolytic degradation, and shielding the chromosome from recognition by the DNA damage-repair system. ${ }^{14}$ Telomeres are essential components that protect against the potential loss of genetic information due to replication flaws. ${ }^{15-18}$ The maintenance of telomere length is strongly associated with long-term human health. Telomere shortening is associated with adverse health outcomes across the lifespan including type 2 diabetes, ${ }^{19}$ cardiovascular diseases, ${ }^{20}{ }^{21}$ cancer, ${ }^{22}{ }^{23}$ Alzheimer's disease,${ }^{24}$ and early mortality. ${ }^{25}{ }^{26}$ Besides being a biomarker for cellular and biologic aging, telomere shortening also reflects the cumulative exposure to oxidative stress and subsequent disease development. ${ }^{1926-28}$

Interestingly, the rate of telomere shortening was found to be accelerated by exposure to environmental chemicals such as tobacco smoke. ${ }^{29}{ }^{30}$ Empirical studies found that smoking was associated with telomere shortening in adults. ${ }^{29} 31$ Compared to adults who have never been exposed to tobacco smoke, the telomere length of exposed adults shrank at a more significant 
rate over time, which suggests tobacco exposure may accelerate the aging process. ${ }^{29}$ Several studies found a dose-response relationship between cumulative lifetime tobacco exposure and telomere length in adults: those with longer exposure to cigarette smoke had shorter telomere lengths. ${ }^{122} 29$

Recent animal studies investigating the potential effect of smoking on telomere length found that developing mouse embryos exposed to cigarette smoke condensate (CSC) and cadmium (a major component in tobacco) resulted in telomere shortening and significant reduction in the cleavage of mouse embryos. ${ }^{29}$ This appeared to be related to chromosomal instability and oxidative damage to embryonic stem cells induced by different levels of stress intensity. ${ }^{30,31}$

In spite of these empirical findings from animal studies, the actual relationship between tobacco exposure in women during pregnancy and the telomere length in their children is still largely unknown. Previous studies on telomere length in children were more focused on the effect of a deprived environment in early childhood. ${ }^{25} 3233$ We still do not know whether maternal smoking during pregnancy leads to telomere shortening in their offspring, and whether this telomere shortening can be a possible mechanism to explain maternal smoking-related detrimental health outcomes in children. Findings from a retrospective study on a small group of disadvantaged US children suggested that infants born to mothers who smoked during pregnancy had shorter telomere lengths, but the study had major limitations in its small sample size and combined measurement in ETS and maternal active smoking. ${ }^{34}$ Meanwhile, another study measuring a newborn's telomere length in the cord blood found a possible relationship between exposure to ETS during pregnancy and shortening of neonatal telomere length. ${ }^{35}$ However, this study did not consider the dosage effect of maternal smoking during pregnancy. ${ }^{35}$ A robust study that considers the effect of the duration of prenatal tobacco exposure and ETS during pregnancy and in 
childhood is still lacking. We have conducted the first such study to investigate the impact of prenatal tobacco exposure on a child's telomere length. We hypothesize that prenatal tobacco exposure is associated with telomere shortening in children.

\section{METHODS}

\section{Study population}

This cross-sectional study examined the association between prenatal tobacco exposure (women actively smoking during pregnancy) and telomere length in children recruited from the Hong Kong Child Health Survey (CHS), a population-based household survey conducted in September 2005 to August 2006 on Chinese children under 15 years of age. The CHS selected households using systematic replicated sampling based on the Register of Quarters, a record of all permanent household quarters maintained by the Hong Kong Census and Statistics Department. ${ }^{36-38}$ A total of 7393 children under 15 years were recruited from all 18 districts of Hong Kong. Among them, 59 children were active smokers who were excluded from this study and 146 children were nonsmokers whose mothers smoked during pregnancy. The exposed group comprised of 98 randomly selected non-smoking children whose mother smoked during pregnancy; the control group comprised of another 98 age- and gender-matched children randomly selected from all non-smoking children whose mothers never smoked during pregnancy.

A previous study identified an effect size of 0.55 between prenatal exposure to tobacco and children's telomere length. ${ }^{34} \mathrm{We}$ adopted a more conservative effect size of 0.41 (75\% of that reported in the above study), which required an effective sample size of 190 children (95 exposed and 95 control). Three additional participants per group were included to give a final sample size of 196 (98 exposed and 98 control), which has at least $80 \%$ statistical power to detect the effect size at a significance level of 0.05 . 


\section{Smoking exposure measures}

Face-to-face interviews were conducted with the parents of all participating children to obtain information on the children's tobacco exposure, general health status, and sociodemographics. The interview was anonymous and did not collect any tracable personal identifiers (e.g. name, address, and identity number) to avoid social desirability bias. If the interview respondent was not certain regarding a certain question, he/she was suggested to discuss with family members.

There were four questions related to the children's tobacco exposure during pregnancy and in childhood, namely "Did the mother smoke during the pregnancy? If yes, for how long?", "Has the mother been exposed to second hand smoking during pregnancy?", "What is the mother's current (or in the past 7 days) smoking habit?", and "What is the mother's current (or in the past 7 days) smoking habit?".

Children's prenatal exposure to tobacco was categorised into: 1) mother did not smoke during pregnancy, 2) mother smoked initially but quit in first trimester of pregnancy, and 3) mother smoked throughout the pregnancy. Children in the last two categories were classified as being exposed to prenatal tobacco. Children's prenatal ETS exposure was categorised into: 1) mother not exposed to ETS during pregnancy, 2) exposed to prenatal ETS for less than $1 \mathrm{~h}$ daily, and 3) exposed to prenatal ETS for at least $1 \mathrm{~h}$ daily. Children's ETS exposure was categorised according to the current smoking status of the parents as: 1) non-smoker, 2) smoked $\leq 5$ cigarettes per day, and 3) smoked > 5 cigarettes per day.

\section{Family socioeconomic status (SES)}

Two key family variables were included to reflect the family SES, family monthly income adjusted for household size and whether the family received Comprehensive Social Security 
Assistance (CSSA), which is a direct cash subsidiary provided by the government to low income families. Family income adjusted for household size was computed using the Organisation for Economic Co-operation and Development (OECD) square-root scale to account for the fact that the household economic need may not be linearly proportional to household size. ${ }^{39}$

\section{DNA extraction and telomere length}

The telomere length was measured in buccal epithelial cells from children participating in the study. Buccal swab samples were collected from each child during a household visit. Genomic DNA was isolated and extracted using the Gentra Puregene Buccal Cell Kit (Qiagen) according to the manufacturer's instruction. The isolated DNA samples were diluted to a concentration of 5 $\mathrm{ng} / \mu \mathrm{L}$ in the buffer solution (10 mM Tris- $\mathrm{HCl}$ and $1 \mathrm{mM}$ EDTA, $\mathrm{pH}$ 8.0) and quantified using a Nanodrop 2000c spectrophotometer (Applied Biosystems).

The relative telomere length was determined by adopting the protocols described by Drury et al. and Cawthon. ${ }^{330}$ For each sample, the telomere length was represented by the relative ratio of the telomere repeat copy number $(\mathrm{T})$ to the single copy gene 36B4 copy number $(\mathrm{S})$. The T/S ratio was determined by quantitative polymerase chain reaction (qPCR) using a 7900HT Thermocycler (Applied Biosystems). It can then be calculated by the formula $\mathrm{T} / \mathrm{S}=2^{(-\mathrm{dCt})}$, where $\mathrm{dCt}$ is the difference in threshold cycle obtained by subtracting the average $36 \mathrm{~B} 4 \mathrm{Ct}$ value from the average telomere $\mathrm{Ct}$ value.

\section{Statistical analysis}

Reliability of the T/S ratio values was tested using the generalized extreme studentized deviate (GESD) test ${ }^{41}$ which is an extension of the Grubbs test for outlier detection that allows testing of multiple potential outliers without inflating type I error. Detected outliers were regarded as 
missing data in the analysis. Missing data was handled using multiple imputations (MI), which is a more robust technique for handling missing-at-random data than mean imputation and complete data analysis. ${ }^{42}$ Imputations were repeated five times in this study and all the analysis results were based on the pooled estimates from the five imputed datasets. ${ }^{42}$

The T/S ratio difference between the exposed (mother smoked during pregnancy) and control (mother did not smoke during pregnancy) groups was examined using paired t-test. The doseresponse relationship between T/S ratio and prenatal tobacco exposure (not exposed, exposed only in first trimester of pregnancy, and exposed throughout pregnancy) was tested using a linear regression model adjusted for the child's age and gender (the crude model). To eliminate the potential confounding effect of SES, family income and CSSA status were additionally controlled (the adjusted model). Similarly, the relationship between T/S ratio and exposure to prenatal ETS and childhood ETS (from paternal and maternal smoking) were tested using crude and adjusted models. Finally, a full model was fitted with all the covariates, including age, gender, family income, CSSA status, prenatal tobacco exposure, prenatal ETS exposure, and childhood ETS exposure from paternal and maternal smoking. In other words, there were four smoking exposure variables in the crude, adjusted, and full models: (i) prenatal tobacco (mother smoking status during pregnancy), (ii) ETS during pregnancy (maternal exposure to ETS), (iii) ETS in childhood due to paternal smoking, and (iv) ETS in childhood due to maternal smoking. Statistical significance was determined using the two-tailed p-value of the regression coefficients. A p-value of less than 0.05 was deemed statistically significant. All analyses were performed in $R$ Statistical Package version 3.2.1 with the package MICE. ${ }^{43}$ 


\section{Ethics Approval}

The study and consent procedures were approved by the ethical committee of the Institutional Review Board of the University of Hong Kong/Hospital Authority Hong Kong West Cluster. Written informed consent was obtained from the parents of each participant.

\section{RESULTS}

A total of 196 children were included in this study (98 exposed, 98 age- and gender-matched control). Telomere lengths of eight children, (three from the control and five from the exposed group) were deemed as outliers by the GESD tests. Detailed descriptions of the respondents' tobacco exposure and family SES are shown in Table 1. The overall mean age of participants was $6.33 \pm 3.85$ years. Among children with direct prenatal tobacco exposure, $90.8 \%$ were exposed throughout pregnancy and $84.7 \%$ were also exposed to ETS during pregnancy. In the control group, $39.8 \%$ of children were exposed to prenatal ETS. Children in the exposed group had much longer ETS exposure during pregnancy and childhood, and $68 \%$ of fathers were current smokers compared to $26.5 \%$ in the control group. Concerning their socioeconomic status, the exposed group had significantly lower family income and more were receiving CSSA than in the control group.

The overall mean telomere length of children participating in this study as represented by the mean $\mathrm{T} / \mathrm{S}$ ratio was $26.96 \pm 11.88$. There was a significant difference in telomere lengths between children in the exposed and control groups. Figure 1 shows the distribution of the T/S ratios among the sampled children. The mean T/S ratio of children with prenatal tobacco exposure was significantly lower than that of children in the control group (24.90 [SD 8.58] in exposed vs. 28.97 [14.15] in control groups; $p=0.016$ ), indicating there was a crude association between prenatal tobacco exposure and the telomere length in children. 
As shown in Table 2, the crude model with child's gender and age as the only covariates indicated a significant and negative association between the child's T/S ratio and the maternal smoking throughout the pregnancy $(\beta=-4.49 ; 95 \% \mathrm{CI}-7.88$ to $-1.10 ; \mathrm{p}<0.01)$. Moreover, the adjusted model with monthly family income and CSSA recipient status as additional covariates confirmed that the T/S ratio was negatively associated with direct tobacco exposure during pregnancy $(\beta=-4.82 ; 95 \%$ CI -8.30 to $-1.35 ; \mathrm{p}<0.01)$. Upon fitting all four exposure variables with the previously included covariates in the full model, we found only direct tobacco exposure due to maternal smoking during pregnancy had a significantly negative association with the child's T/S ratio $(\beta=-6.02 ; 95 \% \mathrm{CI}-11.14$ to $-0.89 ; \mathrm{p}<0.05)$. In all three models, both ETS exposure during pregnancy (mother exposed to ETS) and ETS exposure in childhood related to paternal and maternal smoking did not have any significant association with the child's T/S ratio.

Moreover, prenatal tobacco exposure and children's telomere length had a dose-response relationship. The telomere length of children had a decreasing trend with the increasing duration of direct maternal smoking $(\mathrm{p}=0.02)$, the longer the mother smoked during pregnancy, the shorter the child's telomere length (Figure 2).

\section{DISCUSSION}

\section{Prenatal tobacco exposure and telomere shortening}

This is one of the first studies to demonstrate that smoking during pregnancy causes telomere shortening in children. Our study differed from previous studies ${ }^{34,35}$ in that we considered both the duration of maternal smoking during pregnancy and the influence of ETS during pregnancy and childhood. The study also involved a much larger and representative sample recruited through a population health study. 
A recent study provided evidence of an inverse relationship between foetus's intrauterine tobacco exposure and the cord blood telomere length at birth. ${ }^{35}$ Our findings provide further evidence to support this association, which persisted beyond the newborn period and suggested a longer lasting effect due to prenatal tobacco exposure. Meanwhile, we also found a gradient relationship between the duration of maternal smoking during pregnancy and children's telomere length (Figure 2). However, ETS exposure during pregnancy and childhood did not affect this relationship.

\section{Tobacco smoke exposure and telomere shortening}

Tobacco smoke is a prevalent systemic human mutagens and leads to significant genotoxicity. In utero exposure to tobacco smoke can disrupt the foetus's intrauterine programming and results in the elevated frequencies of hypoxanthine phosphoribosyltransferase (HPRT) mutations, translocations, and DNA strand breaks in newborns. ${ }^{44}$ On the other hand, telomeres are an important biological marker for aging and have been linked with cancer development. ${ }^{45}$ Children diagnosed with neuroblastoma and leukaemia were found to have significant telomere shortening. ${ }^{46}$ Although tobacco smoke exposure has been shown to result in telomere shortening in adults, its effect on telomere length and related potential health implications in children are largely unknown.

Richter and Zglinicki found that oxidative stress-mediated DNA damage was an important determinant of telomere shortening. ${ }^{15284748}$ Laboratory studies investigating the effects of cigarette smoke condensate (CSC) on animal cells and mouse embryos showed that cigarette smoke increased cellular reactive oxygen species load that caused oxidative stress in cells. ${ }^{49}$ Mouse embryonic stem cells exposed to low doses of CSC or cadmium resulted in shortened 
telomere length and DNA damage. ${ }^{50}$ Whole mouse exposed to cigarette smoke increased reactive oxygen species in cells leading to telomere shortening and apoptosis. ${ }^{51}$

The high G-C content of the telomeres renders them susceptible to oxidative stress. Oxidative stress was found to affect the rate of telomere attrition during DNA replication under a range of stress intensities via intensifying the replication problem at different extent. This suggests that cigarette smoke can lead to telomere shortening at different levels of exposure ${ }^{49}$ This could also explain the dose-gradient relationship between the duration of prenatal maternal smoking and child's telomere shortening found in this study.

\section{Environmental tobacco smoking and telomere length}

Although ETS exposure has been linked to adverse effects on child health, ${ }^{52-55}$ we found that ETS exposure during pregnancy and childhood were not significantly associated with telomere shortening or with the relationship between maternal smoking during pregnancy and telomere shortening in children.

\section{Implications of the findings}

Our original findings support the hypothesis that maternal smoking during pregnancy shortens the telomere length in children. As reflected by telomere shortening, in utero exposure to tobacco smoke could have more profound harmful effects than previously expected by affecting the biological programming of foetus, which may lead to adverse long-term outcomes in childhood and adulthood. ${ }^{20-2325} 27$ Our findings demonstrated that harmful effects of prenatal tobacco exposure could be observed well before clinical manifestations. As smoking is still relatively common among pregnant women, these findings have important implications for health care planning and related policy making. ${ }^{56}$ Policy makers should recognize that smoking during 
pregnancy is a key public health issue, and should educate pregnant women and public about the potential harmful effects of prenatal tobacco exposure on the long-term health of children. Successful intervention programs to prevent/reduce smoking among pregnant women should comprise of multiple components with different dimensions. ${ }^{57-59}$ Nonetheless, the power of evidence-based information in convincing pregnant smokers to quit smoking should not be underestimated. The novel finding of telomere shortening in children directly exposed to prenatal tobacco implies that offspring of mothers who smoke might experience additional health risks in early life, and this can be a strong argument to educate pregnant women to persuade them not to smoke during pregnancy. More effective smoking-cessation and counselling programs during pregnancy could be developed based on evidence from this original study.

\section{Limitations of this study}

The findings from this study should be interpreted with several caveats. First, this observational study did not determine the causal relationship between prenatal tobacco exposure and telomere shortening in children, even though the reverse is not likely. Second, parents' tobacco use was not measured by biomarkers and the self-report figures may subject to recall and social desirability bias. To minimize recall bias, we encouraged the mothers to discuss with family members concerning their smoking history if there were not certain for this question. In addition, mothers may have been unwilling to disclose the information for fear of social stigmatization. We attempted to overcome this issue by keeping the questionnaire anonymous without any personal identifiers (e.g. name and identify numbers). Third, parents' telomere lengths were not measured in this study. It would have been helpful to have this information from parents as there may be a high correlation between parents' and children' telomere length. ${ }^{60}$ Nevertheless, the correlation may be partially due to the shared living environment as both the parents and children 
are exposed to similar environmental stressors. Fourth, maternal substance abuse and alcohol use was not measured in this study. Since smoking, alcohol use, and substance abuse was mildly associated, the current effect size could have been overestimated. Nevertheless, this is not very likely to alter our conclusion because of the low prevalence in substance abuse among women in Hong Kong. 6162

\section{Conclusion}

This is the first study to demonstrate a dose-response relationship between the duration of prenatal smoking exposure and telomere length in children. Exposure to ETS during pregnancy and childhood did not affect telomere length in children. As reflected by telomere shortening, prenatal tobacco exposure in children can cause premature aging and increased health risks, which we suggest is entirely preventable. Not smoking during pregnancy or quitting smoking is critical to improving the health outcome of our future generations as prenatal tobacco exposure may affect children's biological programming.

The relationship between tobacco exposure and biological aging could be further studied. First, it would be important to understand how prenatal smoking at various stages of pregnancy affects telomere length in childhood so that the critical point of intervention could be determined. Second, the potential synergistic or additive effects of prenatal and postnatal tobacco exposure on telomere length should also be investigated. Last but not least, longitudinal study on the longterm health outcomes of children with telomere shortening might provide further information concerning the significance of using telomere as a biological marker in children. 


\section{FUNDING}

The original Child Health Survey 2005-2006 was commissioned by the Hong Kong Department of Health. The funding body has no responsibilities in study design, the collection, analysis, and interpretation of data, the writing of the report, and the decision to submit the manuscript.

\section{COMPETING INTERESTS}

The authors declare no competing interest.

\section{ACKNOWLEDGEMENTS}

We would like to thank the families who participated in this study, and the Department of Health for allowing us to use their Child Health Survey data in our study. Dr Brian Chung is the co-first author of this article.

\section{CONTRIBUTOR STATEMENT}

PI and BHYC conceptualised the study, interpreted the data, and critically revised the manuscript. FKWH analysed the data, drafted a part of the manuscript, and critically revised the manuscript. GCFC, WD, WHSW, SLL, and YLL interpreted the data and critically revised the manuscript. DY and WLW conducted the experiment, interpreted the data, and critically revised the manuscript. PYTC and KTST interpreted the data and drafted a part of the manuscript. 


\section{REFERENCES}

1. Salihu HM, Aliyu MH, Pierre-Louis BJ, et al. Levels of Excess Infant Deaths Attributable to Maternal Smoking During Pregnancy in the United States. Matern Child Health J 2003;7:219-27. doi:10.1023/A:1027319517405.

2. Chan S, Lam TH, Wong D, et al. Smoking and Health Survey in Hong Kong Women. Hong Kong Council on Smoking and Health, 2014.

3. Wang X, Tager IB, Van Vunakis H, et al. Maternal smoking during pregnancy, urine cotinine concentrations, and birth outcomes. A prospective cohort study. Int J Epidemiol 1997;26:978-88. doi:10.1093/ije/26.5.978.

4. Abel EL. Smoking During Pregnancy: A Review of Effects on Growth and Development of Offspring. Hum Biol 1980;52:593-625. doi:10.2307/41464571.

5. Hofhuis W, de Jongste JC, Merkus PJFM. Adverse health effects of prenatal and postnatal tobacco smoke exposure on children. Arch Dis Child 2003;88:1086-90. doi:10.1136/adc.88.12.1086.

6. Gilliland FD, Li Y-F, Peters JM. Effects of Maternal Smoking during Pregnancy and Environmental Tobacco Smoke on Asthma and Wheezing in Children. Am J Respir Crit Care Med 2001;163:429-36. doi:10.1164/ajrccm.163.2.2006009.

7. Dong G-H, Wang D, Yang Z-H, et al. Gender-specific differences in effects of prenatal and postnatal environmental tobacco smoke exposure on respiratory symptoms in 23,474 children with and without allergic predisposition: results from 25 districts of northeast China. Int J Environ Health Res 2011;21:173-88. doi:10.1080/09603123.2010.515673.

8. Zheng T, Niu S, Lu B, et al. Childhood Asthma in Beijing, China: A Population-based Case-Control Study. Am J Epidemiol 2002;156:977-83. doi:10.1093/aje/kwf127.

9. Liu J, Leung PWL, McCauley L, et al. Mother's environmental tobacco smoke exposure during pregnancy and externalizing behavior problems in children. Neurotoxicology 2013;34:167-74. doi:10.1016/j.neuro.2012.11.005.

10. Tiesler CMT, Chen C-M, Sausenthaler S, et al. Passive smoking and behavioural problems in children: Results from the LISAplus prospective birth cohort study. Environ Res 2011;111:1173-79. doi:10.1016/j.envres.2011.06.011.

11. Cornelius MD, Day NL. Developmental consequences of prenatal tobacco exposure. Curr Opin Neurol 2009;22:121-25. doi:10.1097/WCO.0b013e328326f6dc.

12. Centre for Disease Control and Prevention. PRAMS and Smoking Data Tables. Secondary PRAMS and Smoking Data Tables 2013. http://www.cdc.gov/prams/datatobaccotables.htm.

13. Gao X, Pan S, Li S, et al. Passive smoking of pregnant women in Guangzhou. Chinese Prev Med 2002;3:224-25.

14. Blackburn EH. Structure and function of telomeres. Nature 1991;350:569-73. doi:10.1038/350569a0.

15. Houben JMJ, Moonen HJJ, van Schooten FJ, et al. Telomere length assessment: Biomarker of chronic oxidative stress? Redox Biol 2008;44:235-46. doi:10.1016/j.freeradbiomed.2007.10.001.

16. Artandi SE, DePinho RA. Telomeres and telomerase in cancer. Carcinogenesis 2010;31:9-18. doi:10.1093/carcin/bgp268.

17. Greider CW, Blackburn EH. Telomeres, telomerase and cancer. Sci Am 1996;274:92-97. 
18. Herborn KA, Heidinger BJ, Boner W, et al. Stress exposure in early post-natal life reduces telomere length: an experimental demonstration in a long-lived seabird. Proc $R$ Soc B 2014;281. doi:10.1098/rspb.2013.3151.

19. Zhu H, Belcher M, van der Harst P. Healthy aging and disease: role for telomere biology? Clin Sci (Lond) 2011;120:427-40. doi:10.1042/CS20100385.

20. Butt HZ, Atturu G, London NJ, et al. Telomere Length Dynamics in Vascular Disease: A Review. Eur J Vasc Endovasc Surg 2010;40:17-26. doi:10.1016/j.ejvs.2010.04.012.

21. Fitzpatrick AL, Kronmal RA, Gardner JP, et al. Leukocyte Telomere Length and Cardiovascular Disease in the Cardiovascular Health Study. Am J Epidemiol 2007;165:14-21. doi:10.1093/aje/kwj346.

22. McGrath M, Wong JYY, Michaud D, et al. Telomere Length, Cigarette Smoking, and Bladder Cancer Risk in Men and Women. Cancer Epidemiol Biomarkers Prev 2007;16:815-19. doi:10.1158/1055-9965.epi-06-0961.

23. Blasco MA. Telomeres and human disease: ageing, cancer and beyond. Nat Rev Genet 2005;6:611-22.

24. Zhang J, Kong Q, Zhang ZM, et al. Telomere Dysfunction of Lymphocytes in Patients With Alzheimer Disease. Cogn Behav Neurol 2003;16:170-76.

25. Shalev I, Entringer S, Wadhwa PD, et al. Stress and telomere biology: A lifespan perspective. Psychoneuroendocrinology 2013;38:1835-42.

doi:10.1016/j.psyneuen.2013.03.010.

26. Bekaert S, de Meyer T, van Oostveldt P. Telomere Attrition as Ageing Biomarker. Anticancer Res 2005;25:3011-21.

27. Bojesen SE. Telomeres and human health. J Int Med 2013;274:399-413. doi:10.1111/joim.12083.

28. von Zglinicki T. Oxidative stress shortens telomeres. Trends Biochem Sci 2002;27:33944. doi:10.1016/S0968-0004(02)02110-2.

29. Valdes AM, Andrew T, Gardner JP, et al. Obesity, cigarette smoking, and telomere length in women. Lancet 2005;366:662-64. doi:10.1016/S0140-6736(05)66630-5.

30. Cherkas LF, Hunkin JL, Kato BS, et al. THe association between physical activity in leisure time and leukocyte telomere length. Arch Intern Med 2008;168:154-58. doi:10.1001/archinternmed.2007.39.

31. Morlá M, Busquets X, Pons J, et al. Telomere shortening in smokers with and without COPD. Eur Respir J 2006;27:525-28. doi:10.1183/09031936.06.00087005.

32. Entringer S, Epel ES, Lin J, et al. Maternal psychosocial stress during pregnancy is associated with newborn leukocyte telomere length. Am J Obstet Gynecol 2013;208:134.e1-34.e7. doi:10.1016/j.ajog.2012.11.033.

33. Drury SS, Theall K, Gleason MM, et al. Telomere length and early severe social deprivation: linking early adversity and cellular aging. Mol Psychiatry 2012;17:719-27.

34. Theall KP, McKasson S, Mabile E, et al. Early Hits and Long-Term Consequences: Tracking the Lasting Impact of Prenatal Smoke Exposure on Telomere Length in Children. Am J Public Health 2013;103:S133-S35. doi:10.2105/ajph.2012.301208.

35. Salihu HM, Pradhan A, King L, et al. Impact of intrauterine tobacco exposure on fetal telomere length. Am J Obstet Gynecol 2015;212:205.e1-05.e8. doi:10.1016/j.ajog.2014.08.026.

36. Department of Health. Child Health Survey 2005-2006. Hong Kong: Department of Health, HKSAR Government, 2014. 
37. Lee SL, Cheung YF, Wong HSW, et al. Chronic health problems and health-related quality of life in Chinese children and adolescents: a population-based study in Hong Kong. BMJ Open 2013;3:e001183. doi:10.1136/bmjopen-2012-001183.

38. Ho MH, Lee SL, Wong WH, et al. Prevalence of self-reported food allergy in Hong Kong children and teens - a population survey Asian Pac J Allergy Immunol 2012;30:275 - 84.

39. OECD. What are equivalence scales. Paris: The Organisation for Economic Co-operation and Development 2013.

40. Cawthon RM. Telomere measurement by quantitative PCR. Nucleic Acids Res 2002;30:e47-e47.

41. Rosner B. Percentage points for a generalized ESD many-outlier procedure. Technometrics 1983;25:165-72.

42. Rubin DB. Multiple imputation for nonresponse in surveys. New York: John Wiley \& Sons, 2004.

43. R: A Language and Environment for Statistical Computing [program]. 3.2.1 version. Vienna, Austria: R Foundation for Statistical Computing, 2015.

44. DeMarini DM. Genotoxicity of tobacco smoke and tobacco smoke condensate: a review. Mutat Res Rev Mutat Res 2004;567:447-74.

45. Tabori U, Nanda S, Druker H, et al. Younger Age of Cancer Initiation Is Associated with Shorter Telomere Length in Li-Fraumeni Syndrome. Cancer Res 2007;67:1415-18. doi:10.1158/0008-5472.can-06-3682.

46. Binz N, Shalaby T, Rivera P, et al. Telomerase inhibition, telomere shortening, cell growth suppression and induction of apoptosis by telomestatin in childhood neuroblastoma cells. Eur J Cancer 2005;41:2873-81. doi:10.1016/j.ejca.2005.08.025.

47. Richter T, von Zglinicki T. A continuous correlation between oxidative stress and telomere shortening in fibroblasts. Exp Gerontol 2007;42:1039-42.

48. Oikawa S, Kawanishi S. Site-specific DNA damage at GGG sequence by oxidative stress may accelerate telomere shortening. FEBS Lett 1999;453:365-68. doi:10.1016/S00145793(99)00748-6.

49. Huang J, Okuka M, McLean M, et al. Telomere susceptibility to cigarette smoke-induced oxidative damage and chromosomal instability of mouse embryos in vitro. Redox Biol 2010;48:1663-76.

50. Huang J, Okuka M, Lu W, et al. Telomere shortening and DNA damage of embryonic stem cells induced by cigarette smoke. Reprod Toxicol 2013;35:89-95.

51. Huang J, Okuka M, McLean M, et al. Effects of cigarette smoke on fertilization and embryo development in vivo. Fertil Steril 2009;92:1456-65.

52. Simons E, To T, Moineddin R, et al. Maternal second-hand smoke exposure in pregnancy is associated with childhood asthma development. J Allergy Clin Immunol Pract 2014;2:201-07. e3.

53. Zhou S, Rosenthal DG, Sherman S, et al. Physical, Behavioral, and Cognitive Effects of Prenatal Tobacco and Postnatal Secondhand Smoke Exposure. Curr Probl Pediatr Adolesc Health Care 2014;44:219-41.

54. Carlsen K-H, Carlsen KCL. Respiratory effects of tobacco smoking on infants and young children. Paediatr Respir Rev 2008;9:11-20.

55. Thacher JD, Gruzieva O, Pershagen G, et al. Pre-and postnatal exposure to parental smoking and allergic disease through adolescence. Pediatrics 2014;134:428-34. 
56. Caleyachetty R, Tait CA, Kengne AP, et al. Tobacco use in pregnant women: analysis of data from Demographic and Health Surveys from 54 low-income and middle-income countries. Lancet Global Health 2014;2:e513-e20.

57. Greaves L, Poole N, Okoli CTC, et al. Expecting to Quit: A best practices review of smoking cessation interventions for pregnant and post-partum women. 2nd ed. Vancouver: British Columbia Centre of Execellence for Women's Health, 2011.

58. Hettema JE, Hendricks PS. Motivational interviewing for smoking cessation: A metaanalytic review. J Consult Clin Psychol 2010;78:868-84. doi:10.1037/a0021498.

59. Coleman T. Reducing harm from tobacco smoke exposure during pregnancy. Birth Defects Res C Embryo Today 2008;84:73-79. doi:10.1002/bdrc.20115.

60. Broer L, Codd V, Nyholt DR, et al. Meta-analysis of telomere length in 19713 subjects reveals high heritability, stronger maternal inheritance and a paternal age effect. Eur $J$ Hum Genet 2013;21:1163-68.

61. Hong Kong Census and Statistics Department. Drug Abuse Situation in Hong Kong in 2012. Hong Kong Census and Statistics Department, 2013.

62. Department of Health. Action Plan to Reduce Alcohol-related Harm in Hong Kong. Hong Kong: Department of Health, 2013. 
Table 1. Characteristics of the study participants

\begin{tabular}{|c|c|c|c|c|}
\hline & Overall & Exposed & Control & p-value \\
\hline Gender, n (\%) & & & & $-^{\mathrm{a}}$ \\
\hline Female & $98(50.0)$ & $49(50.0)$ & $49(50.0)$ & \\
\hline Male & $98(50.0)$ & $49(50.0)$ & $49(50.0)$ & \\
\hline Mean (SD) age (years), & $6.33(3.85)$ & $6.33(3.86)$ & $6.33(3.86)$ & $-{ }^{\mathrm{a}}$ \\
\hline $\mathrm{T} / \mathrm{S}$ ratio, mean $(\mathrm{SD})$ & $26.96(11.88)$ & $24.90(8.58)$ & $28.97(14.15)$ & 0.02 \\
\hline \multicolumn{5}{|l|}{ Prenatal exposure to tobacco, n (\%) } \\
\hline None & $98(50.0)$ & $0(0.0)$ & $98(100.0)$ & \\
\hline In first trimester of pregnancy & $9(4.6)$ & $9(9.2)$ & $0(0.0)$ & \\
\hline Throughout pregnancy & $89(45.4)$ & $89(90.8)$ & $0(0.0)$ & \\
\hline Prenatal ETS exposure, n (\%) & & & & $<0.001$ \\
\hline None & $74(37.8)$ & $15(15.3)$ & $59(60.2)$ & \\
\hline Exposed to $<1$ hour per day & $92(46.9)$ & $55(56.1)$ & $37(37.8)$ & \\
\hline Exposed to $\geq 1$ hour per day & $29(14.8)$ & $27(27.6)$ & $2(2.0)$ & \\
\hline Missing & $1(0.5)$ & $1(1.0)$ & $0(0.0)$ & \\
\hline Current smoking status of mother, $n(\%)$ & & & & $<0.001$ \\
\hline Non-smoker & $116(59.2)$ & $25(25.5)$ & $91(92.9)$ & \\
\hline Smoked $\leq 5$ cigarettes per day & $54(27.6)$ & $48(49.0)$ & $6(6.1)$ & \\
\hline Smoked $>5$ cigarettes per day & $22(11.2)$ & $22(22.4)$ & $1(1.0)$ & \\
\hline Missing & $4(2.0)$ & $3(3.1)$ & $0(0.0)$ & \\
\hline Current smoking status of father, n (\%) & & & & $<0.001$ \\
\hline Non-smoker & $103(52.6)$ & $31(31.6)$ & $72(73.5)$ & \\
\hline Smoked $\leq 5$ cigarettes per day & $43(21.9)$ & $29(29.6)$ & $14(14.3)$ & \\
\hline Smoked $>5$ cigarettes per day & $46(23.5)$ & $35(35.7)$ & $11(11.2)$ & \\
\hline Missing & $4(2.0)$ & $3(3.1)$ & $1(1.0)$ & \\
\hline Adjusted family income, mean (SD), HKD & $19,770(13,490)$ & $17,300(9,960)$ & $22,110(15,850)$ & 0.11 \\
\hline CSSA, n $(\%)$ & & & & 0.09 \\
\hline Yes & $10(5.1)$ & $8(8.2)$ & $2(2.0)$ & \\
\hline No & $165(84.2)$ & 77 (78.6) & $88(89.8)$ & \\
\hline Missing & $21(10.7)$ & $13(13.3)$ & $8(8.2)$ & \\
\hline
\end{tabular}

${ }^{\mathrm{a}}$ Not available due to study design 
Table 2. Association between exposure to tobacco and T/S ratio

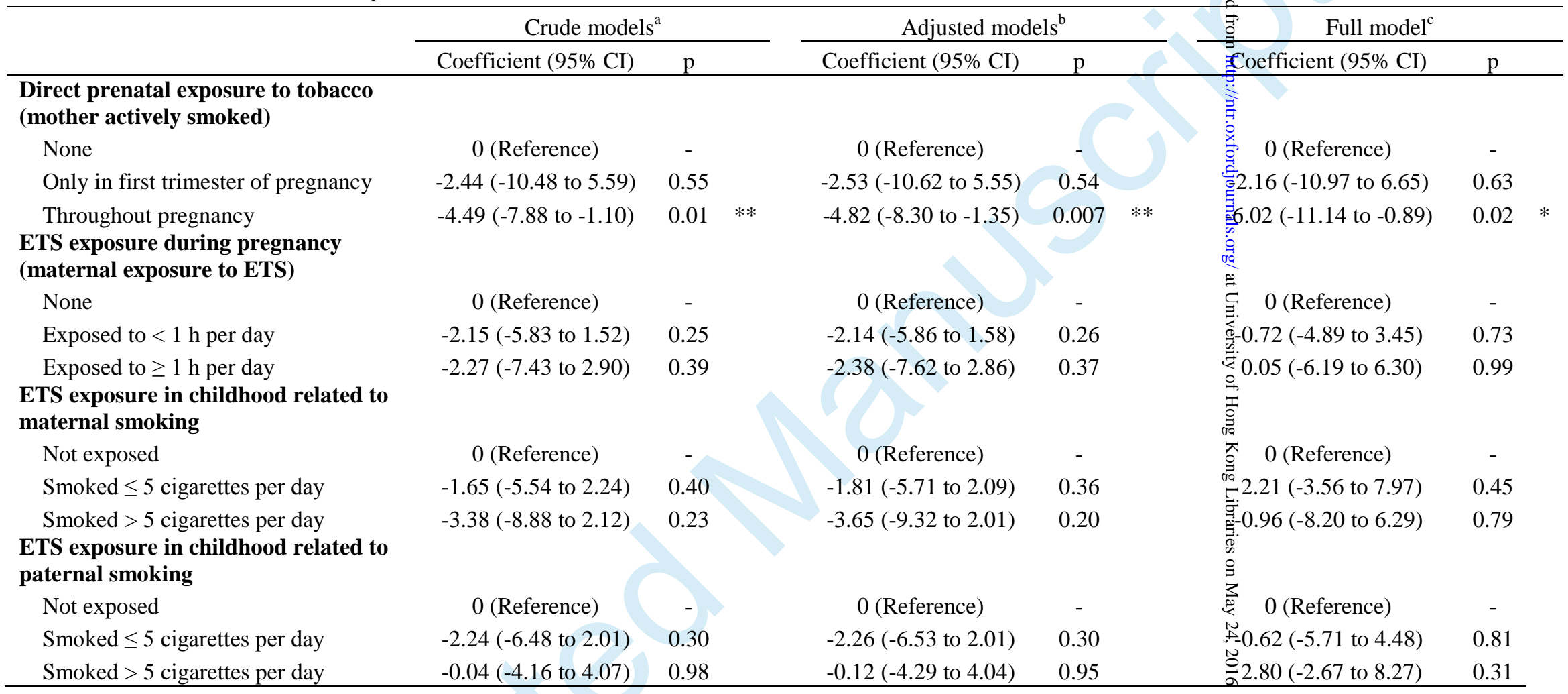

${ }^{a}$ Crude models were constructed for each of the four exposure variables with the adjustment of children's age and gender

bAdjusted models controlled family monthly income and CSSA status for each exposure variable, in addition to children's age and gender

${ }^{c}$ The full model included all exposure variables, children's age, gender, adjusted family monthly income, and CSSA status

** $\mathrm{P}<0.01 ; * \mathrm{P}<0.05$ 
Figure 1. Distribution of T/S ratio by direct prenatal exposure to tobacco

Exposed children whose mothers smoked during pregnancy; control children whose mothers did not smoke d产ring pregnancy. 
Figure 2. Mean T/S ratio according to different levels of prenatal direct exposure of tobacco

Error bar shows the 95\% CI. 
Figure 1

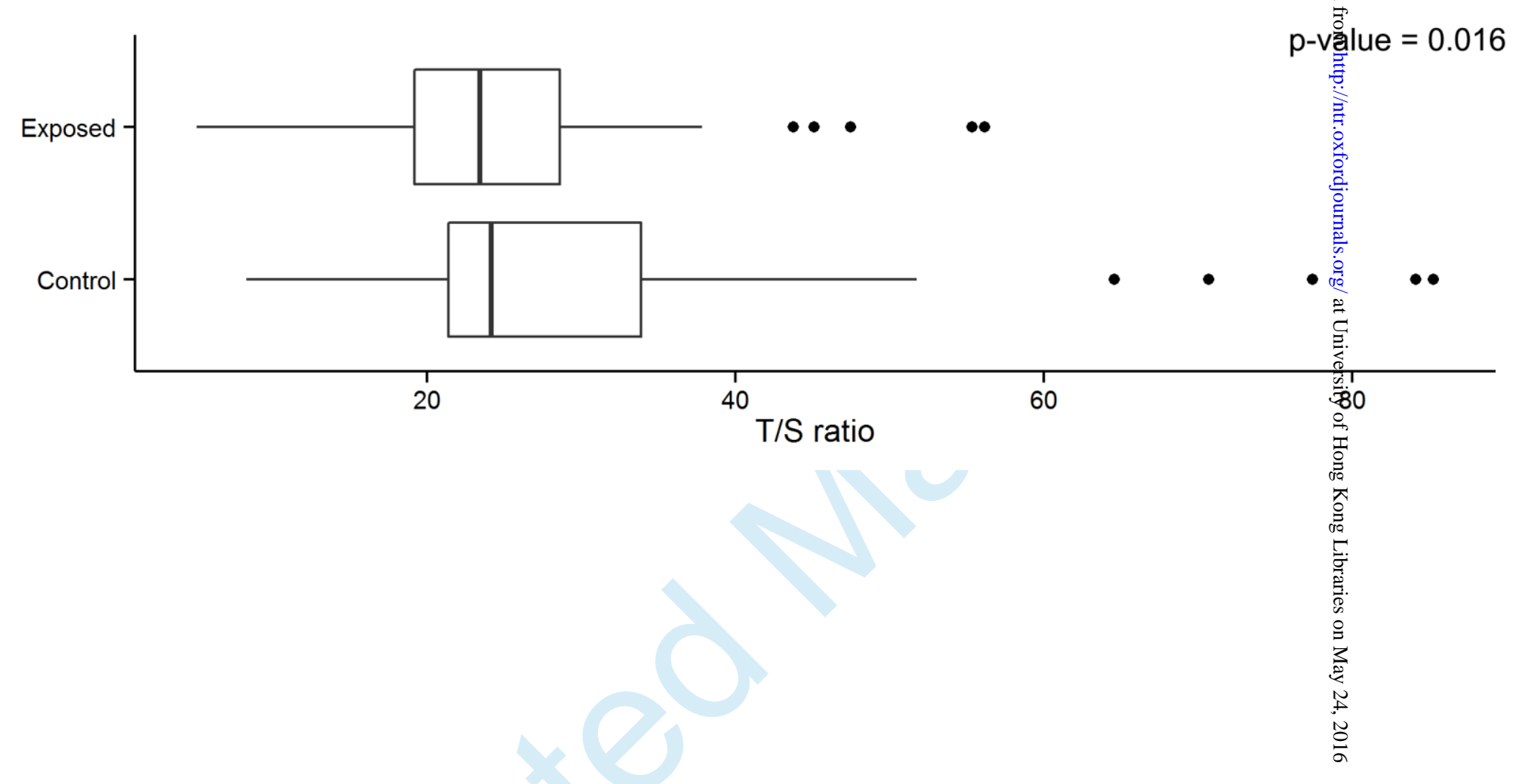


Figure 2

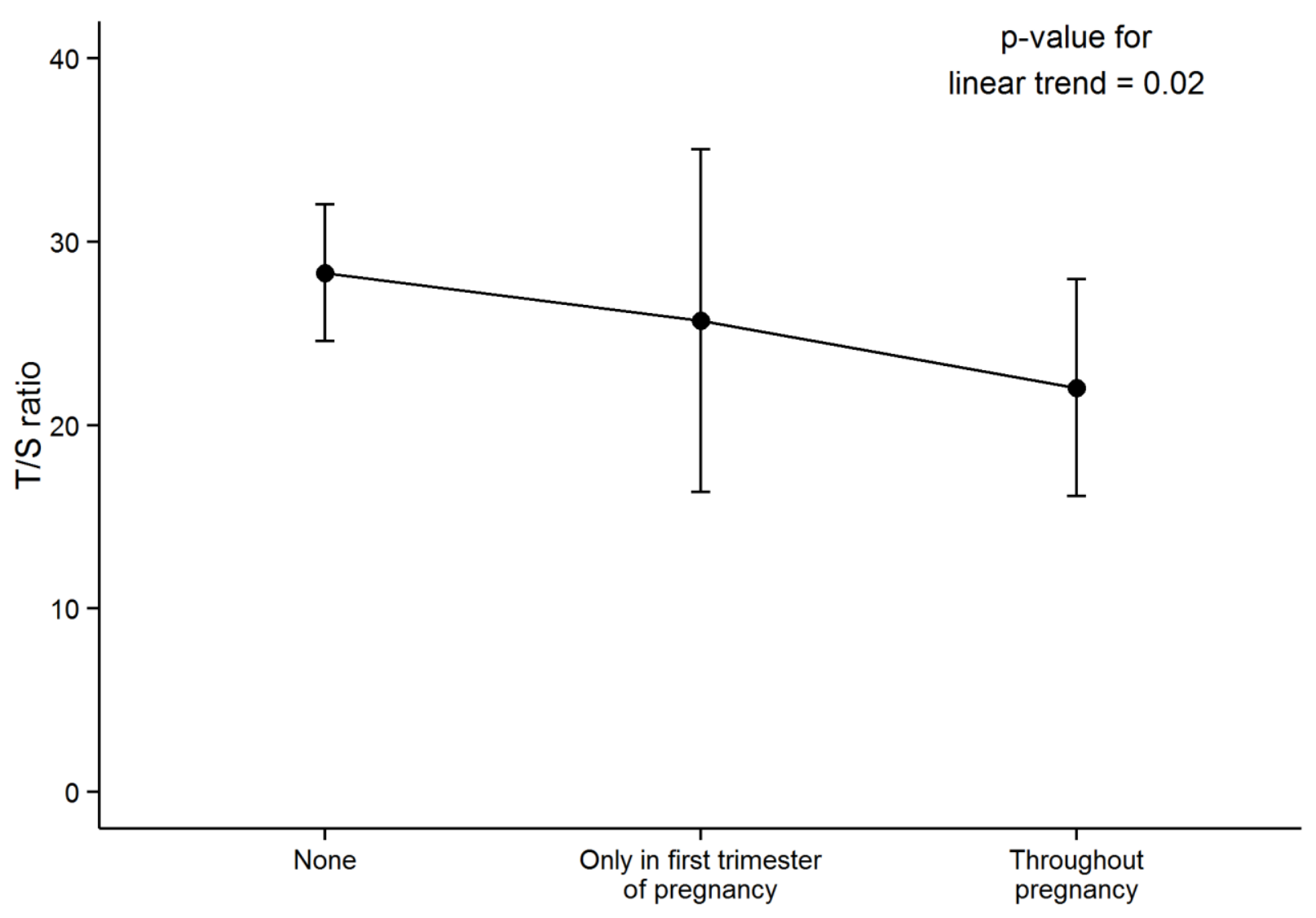

Direct prenatal exposure to tobacco 